\title{
Biochemical Bone Markers as Predictors of Excessive Bone Turnover Suppression during Osteoporosis Treatment with Bisphosphonates
}

Branka Kovacev-Zavisic ${ }^{1}$, Tijana $_{\text {Icin }}{ }^{*}$, Jovanka Novakovic-Paro' ${ }^{1}$, Milica Medic-Stojanoska1, Milena Mitrovic ${ }^{1}$, Dragana Tomic - Naglic ${ }^{1}$, Ivana Bajkin ${ }^{1}$, Nikola Curic ${ }^{2}$, Milan Dolga ${ }^{3}$ and Nemanja Kovacev ${ }^{4}$

${ }^{1}$ Clinic of Endocrinology, Diabetes and Metabolic Disorders, Clinical Centre of Vojvodina, Novi Sad, Serbia

${ }^{2}$ Center of Laboratory Medicine, Clinical Centre of Vojvodina, Novi Sad, Serbia

${ }^{3}$ Smart Line agency, Trg Republike 13, Novi Sad, Serbia

${ }^{4}$ Clinic of Orthopaedic surgery and Traumatology, Clinical Center of Vojvodina, Novi Sad, Serbia

\begin{abstract}
Background: It should be decided before the introduction of osteoporosis therapy whether to start the therapy with the inhibitor of resorption or with the stimulator for bone formation. The decision should also be made during the therapy with antiresorptives regarding the appropriate time to stop the therapy and start the therapy with stimulators for bone formation. Assuming that normal or reduced levels of bone metabolism is limiting factor for initiation of antiresorptive therapy, and thereafter the appropriate parameter for the decision to stop antiresorptive therapy and start with stimulators of bone metabolism, we believe that the determination of bone markers should be compulsory and an integral part of the diagnostic procedure. The aim of this study was to determine whether antiresorptive therapy leads to satisfactory or excessive suppression of bone metabolic activity depending on the initial values of bone markers
\end{abstract}

Methods: We performed a prospective longitudinal study of 178 postmenopausal women with osteoporosis. We were following the values of osteocalcin, beta-crosslaps before the introduction of bisphosphonates therapy and after three months during therapy.

Results: The results speak in favor of decreased bone resorption during antiresorptive therapy and that the value of bone markers during antiresorptive therapy may be predicted depending on the initial values. If we add osteocalcin and $\beta C T x$ values before the therapy to the equation: $-0.041^{*} \mathrm{OC}-0.003^{*} \beta C T x+2.983$, patients having result $>0$, will have excessive suppression of bone resorption during the therapy. If we add values to the equation: $-0.054^{\star} \mathrm{OC}-0.001^{\star} \beta C T x+2.075$, patients having result $>0$, will have excessive suppression of entire bone remodeling during the therapy.

Conclusion: We suggest that osteoporosis patients who are predicted to have excessive suppression of entire bone remodeling during bisphosphonate therapy, should be mainly treated with stimulators for bone formation or possibly with medications with dual mode of action.

Keywords: Bisphosphonates; Osteoporosis; Bone markers; Bone turnover

\section{Introduction}

Osteoporosis is systemic skeletal disorder characterized by low bone mass and micro architectural deterioration of bone tissue with a consequent increase in susceptibility to fracture [1]. It occurs when various factors cause the loss of physiological connection between bone formation and bone resorption with resorption predominance so the quantity of newly formed bone is insufficient to replace the quantity of resorbed bone to the full extent [2].

After diagnosing and making decisions about starting the therapy of osteoporosis, some of national guides recommend the control of biochemical markers of bone metabolism before the therapy and during it for the purpose of following the effects of the very therapy [3-5].

In addition to the most commonly used bisphosphonate therapy, teriparatide (synthetic parathyroid hormone) has appeared in the last few years as the only potent medicine which has to be effective by increasing bone formation, that is to increase the activity of osteoblast as opposite to antiresorptive therapy which decreases bone resorption, i.e., reduces the activity of osteoclast [6-8].

The potency of antiresorptive therapy has been proved many times and its effects on biochemical markers of bone metabolism have been shown in terms of decreasing bone degradation [9-11].

Having in mind the therapy which would have effect on increasing the activity of osteoblast, it should be decided before the introduction of osteoporosis therapy whether to start the therapy with the inhibitor of resorption or with the stimulator for bone formation. The decision should also be made during the therapy with antiresorptives regarding the appropriate time to stop the therapy and start the therapy with stimulators for bone formation.

Biochemical markers of bone metabolism such as osteocalcin (the marker referring to bone formation and bone remodeling)

*Corresponding author: Tijana Icin, Clinic of endocrinology, diabetes and metabolic disorders, Clinical Centre of Vojvodina, Hajduk Veljkova 1-3, 21000 Novi Sad, Serbia, Tel: +381658610500; Fax: +38121521469; E-mail: tijana.icin@gmail.com

Received January 18, 2013; Accepted February 19, 2013; Published February 21, 2013

Citation: Kovacev-Zavisic B, Icin T, Novakovic-Paro J, Medic-Stojanoska M Mitrovic M, et al. (2013) Biochemical Bone Markers as Predictors of Excessive Bone Turnover Suppression during Osteoporosis Treatment with Bisphosphonates. J Mol Biomark Diagn S10: 001. doi:10.4172/2155-9929.S10-001

Copyright: (C) 2013 Kovacev-Zavisic B, et al. This is an open-access article distributed under the terms of the Creative Commons Attribution License, which permits unrestricted use, distribution, and reproduction in any medium, provided the original author and source are credited 
Citation: Kovacev-Zavisic B, Icin T, Novakovic-Paro J, Medic-Stojanoska M, Mitrovic M, et al. (2013) Biochemical Bone Markers as Predictors of Excessive Bone Turnover Suppression during Osteoporosis Treatment with Bisphosphonates. J Mol Biomark Diagn S10: 001. doi:10.4172/2155-9929.S10-001

and $\beta$-CrossLaps ( $\beta$ CTx, bone resorption marker) provide precise information about current bone metabolical activity or bone turnover [12-14].

Most authors from the field of osteology agree that patients who have more rapid bone metabolism can expect to have benefits from antiresorptive therapy if values of biochemical markers normalize and 'enter' the range of expected values. When biochemical markers have values which are below the reference values then we have to deal with over-treated patients or excessive suppression of bone metabolism [1517].

Antiresorptive therapy increases BMD by slowing down the process of bone resorption, decreasing the number of osteoclasts and their activities, reducing the number of active multicellular units which reduce the total size of resorptive area. Already existing cavitations made by the process of resorption that has already started, are filled in during the first few months of antiresorptive therapy due to finishing the cycle of remodeling in favor of osteoblast activity.

It is thought that this process is responsible for the highest increase of BMD in the first few months of antiresorptive therapy. However, during antiresorptive therapy, slow and inadequate process of remodeling has seemed to occur embodied in forming "less valuable" bone with reduced strength. Bone formation under the influence of antiresorptive therapy causes decreased diversity of bone trabeculas which are not able any more to prevent the expansion of microfractures. In 1999, Tasuku Mashiba et al. reached the conclusion that accumulation of micro bone damages and reduction of bone strength occur on the ribs of dogs treated by risendronate or alendronate [18]. Few years later, the cases of atypical fractures of invertebrate localizations were reported by various authors.

Most of these patients had excessive remodeling suppression which was hystomorphometrically confirmed by some patients' bone biopsies. It was noticed that these patients had suppression of bone resorption with a number of giant osteoclasts, noticeable suppression of bone formation accompanied with diminished or non-existing osteoblast activity and complete absence of bone matrix synthesis. These effects were attributed to long-term and excessive suppression of bone remodeling by receiving bisphosphonates or the combination of bisphosphonates and estrogens or Glucocorticoids [19,20]. In 2010, Andrea Giusti et al. [21], followed by Tero Yli Kyyny [22] in 2011 published the literature review based on the case studies or series of case studies of atypical fractures during antiresorptive therapy publicized from 2005 to 2010/11. Duration of bisphosphonate therapy of these patients was from two to ten years. In most cases described, the values of biochemical markers were decreased or its value was on the bottom line of normal values, especially in the case of bone formation markers (osteocalcin and PINP). Deficiency of all these published cases was based on the fact that bone biochemical markers were measured after fractures had occurred so there had been no evidence referring to the degree of bone turnover before fractures or during antiresorptive therapy. However, in all the cases where biopsy of iliac bone was done, there was conspicuous remodeling suppression accompanied with absolute lack of tetracyclin coloring as an indicator of bone remodeling [21,22].

Above facts suggest that in the last few years, excessive suppression of bone turnover has been considered as potentially harmful effect of antiresorptive therapy. It can be logically concluded that in clinical work, we need to make distinction between those patients who will have desirable suppression of bone remodeling and the patients who will have excessive suppression of bone remodeling as a response to introducing antiresorptive therapy.

This research is designed and guided by the author's wish to make contribution to existing information about the conduct of biochemical bone markers during the antiresorptive treatment of osteoporosis. Its aim is to indicate changes of biochemical bone markers in patients with low values of bone resorption, bone forming and complete bone remodeling.

\section{Materials and Methods}

\section{Materials}

Research, in a form of prospective longitudinal study, has been carried out at the Clinic for Endocrinology, Diabetes and Diseases of Metabolism, Clinical Center of Vojvodina, Novi Sad from 2009 to 2011. It comprised 178 women participants whose diagnosis of osteoporosis had been verified on the basis of the criteria established by the WHO (World Health Organization). All clinical study participants were subjected to bisphosphonate therapy of osteoporosis according to current National Guidebook on Fighting Osteoporosis [23]. All clinical study participants signed an informed consent document to ensure that they would participate in the research. Participants were included in the research by using the following criteria: patients with diagnosed osteoporosis, patients who did not undergo osteoporosis treatment (with bisphosphonates, hormone replacement therapy (HRT), selective estrogen receptor modulators (SERMs), calcitonin, etc.) except the supplementation with vitmain $\mathrm{D}$ and calcium. Criteria by which patients were not included in the research were as follows: existence of comorbidity having repercussions on bone density (malignant diseases, diseases of thyroid and/or parathyroid glands, hypercorticism, chronic kidney disease, malabsorption syndrome, etc.), the therapy of osteoporosis which was earlier introduced (bisphosphonates, HST, SERMs, calcitonin, etc.). Criteria which were used for the purpose of excluding the patients from the research were as follows: uncooperative behavior (irregular medicine taking, avoiding medical check-ups, etc.), progression of some diseases or existence of certain states which were acknowledged during research threatening to affect bone metabolism; patients' unwillingness to participate in the research.

\section{Methods}

Before starting the therapy, detailed anamnesis had been taken and medical examination had been done at the first meeting with a research participant. Blood samples for testing were then taken so that the next parameters could be established: osteocalcin level (OC1, bone formation parameter), $\beta \mathrm{CTx}$ (CL1, bone degradation parameter). After that the therapy of osteoporosis, according to the National Guidebook on Fighting Osteoporosis had been applied. During the next meeting with the patient, appointed three months after the first one, blood samples were taken again for the purpose of establishing levels of: osteocalcin and $\beta \mathrm{CTx}$.

Parameters of bone remodeling activity, such as osteocalcin and $\beta C T x$ levels, were established from the patients' serum by applying standard chemiluminiscent immunometric method based on the use of apparatus "ALECSYS" in the Center for Laboratory Medicine, the Clinical Center of Vojvodina, Novi Sad. The range of reference values for osteocalcin were $12-41 \mathrm{ng} / \mathrm{ml}$ and the range of reference values for $\beta$-crosslaps were $162-436 \mathrm{pg} / \mathrm{ml}$.

Division into groups was done due to the fact that $\beta$ CTx was appropriate marker for tracking bone resorption and osteocalcin was 
Citation: Kovacev-Zavisic B, Icin T, Novakovic-Paro J, Medic-Stojanoska M, Mitrovic M, et al. (2013) Biochemical Bone Markers as Predictors of Excessive Bone Turnover Suppression during Osteoporosis Treatment with Bisphosphonates. J Mol Biomark Diagn S10: 001. doi:10.4172/2155-9929.S10-001

the appropriate bone marker both for bone formation and entire bone remodeling.

\section{Mathematical and statistical analysis}

Mathematical and statistical analysis was used to examine the difference between the groups of research participants.

Group 1a: those patients, whose $\beta C T x$ values, during antiresorptive therapy, were below the range of reference values, were said to have excessive suppression of bone resorption and Group 2a: other research participants.

It was then used for establishing the difference between subgroups of research participants.

Group 1b: those patients whose $\beta C T x$ values and osteocalcin levels were below the range of reference values during the therapy were said to have excessive suppression of entire bone remodeling and Group 2b: other research participants.

Having known that data were parametric (values of osteocalcin and $\beta \mathrm{CTx}$ ), parametric procedure according to the methodology of Milan Dolga from the Agency Smart Line, Novi Sad, Serbia was applied: Multivariate procedures MANOVA and discriminative analysis were used in the analysis. Univariate procedures, such as ANOVA and $t$-test, were applied. The calculation of discrimination coefficient separated specific features of subsamples and excluded them from further processing. In other words, data reduction was performed. Homogeneity of subsamples and their distance were shown and the Cluster analysis was applied for the purpose of studying the phenomenon in the best possible way and making precise and reliable estimation and prediction. This procedure presented descriptive parameters, mean values $(x)$, standard deviation $(\mathrm{Sd})$, minimal and maximal values, coefficients of variation $(\mathrm{Cv})$, confidence interval $(\mathrm{CI})$, asymmetry measures - Skewness, flattening measures- Kurtosis and Kolmogorov-Smirnov test results.

\section{Results}

Average age of patients, average age referring to menarche, onset of menopause, duration of menopause, duration of childbearing period, duration of lactation and number of children is shown in table 1. Prevalence of positive family anamnesis, the influence of smoking, the existence of fragility fractures and height loss $>4 \mathrm{~cm}$ is shown in table 2 .

On examining lumbar spine and hip, osteoporosis has been diagnosed by DXA medical examination in 82 research participants (46\%) while osteoporosis has been verified by RTG of thoracic-lumbar spine in other 96 participants (54\%).

There is no significant statistical difference between biochemical bone markers before the therapy and during it depending on the type of bisphosphonate therapy applied (Table 3 ).

Average values of osteocalcin and $\beta \mathrm{CTx}(\mathrm{OC} 1$ and $\mathrm{CL} 1$ - before the therapy; OC2 and CL2 - during the therapy) is shown in table 4.

\section{Excessive suppression of bone resorption}

Based on reference values for osteocalcin and $\beta C T x$, examined group has been divided into two subgroups: Group 1a, composed of examinees whose $\beta C T x$ values, during bisphosphonate therapy, has shown values which are below the bottom line of reference values for women in fertile period (excessively suppressed bone resorption) and Group 2a, composed of other participants. Central and dispersive parameters and asymmetry and flattening measures of laboratory parameters during the therapy in groups are shown in table 5.

Based on values $\mathrm{p}=0.000$ (analysis MANOVA) and $\mathrm{p}=0.000$ (discriminative analysis) from tables 6 and 7, we can conclude that significant statistical difference between groups of examinees is such

\begin{tabular}{|l|c|c|c|c|}
\hline $\mathbf{N}=1 \mathbf{1 7}$ & $\bar{x}$ & Sd & Min & Max \\
\hline Age of patients (age) & 62,49 & 9,75 & 30 & 84 \\
\hline Menarche (age) & 13,82 & 1,58 & 10 & 20 \\
\hline The onset of menopause (age) & 47,60 & 5,05 & 30 & 58 \\
\hline Duration of childbearing period (age) & 33,78 & 5,07 & 13 & 45 \\
\hline Duration of menopause (age) & 14,89 & 9,32 & 0 & 42 \\
\hline Duration of lactation (months) & 10,76 & 11,57 & 0 & 72 \\
\hline Parity (number of children) & 1,399 & 0,97 & 0 & 6 \\
\hline
\end{tabular}

Table 1: Average age of patients, average age referring to menarche, onset of menopause, duration of menopause, duration of childbearing period, duration of lactation and number of children of 178 clinical study participants receiving bisphosphonate therapy.

\begin{tabular}{|l|l|l|l|l|}
\hline $\mathbf{N = 1 7 8}$ & YES & NO & YES (\%) & NO (\%) \\
\hline $\begin{array}{l}\text { Family } \\
\text { anamnesis }\end{array}$ & 52 & 126 & 24,16 & 75,84 \\
\hline $\begin{array}{l}\text { Smoking } \\
\text { Fragility } \\
\text { fractures }\end{array}$ & 45 & 133 & 32,02 & 67,98 \\
\hline $\begin{array}{l}\text { Height loss } \\
>4 \mathrm{~cm}\end{array}$ & 57 & 135 & 25,28 & 74,72 \\
\hline
\end{tabular}

Table 2: Prevalence of positive family anamnesis, the influence of smoking, the existence of fragility fractures and height loss $>4 \mathrm{~cm}$ of 178 research participants receiving bisphosphonate therapy.

\begin{tabular}{|c|c|c|c|c|}
\hline \multirow{2}{*}{$\mathrm{N}=178$} & \multicolumn{4}{|c|}{ Bisphosphonates } \\
\hline & $10 \mathrm{mg}$ A / D & $70 \mathrm{mg} \mathrm{A} / \mathrm{W}$ & $150 \mathrm{mg} \mathrm{I} / \mathrm{M}$ & Total \\
\hline $\mathrm{N}$ & 97 & 56 & 25 & 178 \\
\hline$\%$ & 54,49 & 31,46 & 14,05 & 100 \\
\hline
\end{tabular}

A - alendronate, I - ibandronate, D - daily, W - weekly, M - monthly

There is no significant statistical difference between biochemical bone markers before the therapy and during it depending on the type of bisphosphonate therapy applied

Table 3: Prevalence of different modes of antiresorptive therapy in participants.

\begin{tabular}{|c|c|c|c|c|c|c|c|}
\hline $\mathbf{N = 1 7 8}$ & $\bar{x}$ & Sd & SE & Min & Max & Cv & CI \\
\hline OC1 & 31.79 & 12.76 & .96 & 4.1 & 78.9 & 40.15 & $\begin{array}{c}29.90- \\
33.67\end{array}$ \\
\hline CL1 & 551.23 & 268.11 & 20.09 & 18.8 & 1290.0 & 48.64 & $\begin{array}{c}511.57- \\
590.90\end{array}$ \\
\hline OC2 & 20.96 & 9.86 & .74 & 3.0 & 63.6 & 47.06 & $\begin{array}{c}19.50- \\
22.42\end{array}$ \\
\hline CL2 & 200.89 & 144.31 & 10.82 & 10.0 & 712.6 & 71.83 & $\begin{array}{c}179.54- \\
222.24\end{array}$ \\
\hline
\end{tabular}

Assymetry and flattening values ranging from -.04 to .04 were not discussed. Minimal (min) i maximal (max) values of bone markers of participants compared to the sample of 178 participants indicate that the values are within the expected range. Larger values of coefficient of variation denote that there is heterogeneity compared to the sample of 178 participants in terms of: OC1 (40.15), CL1 (48.64), OC2 (47.06), CL2 (71.83). There is significant statistical difference between starting values and therapeutic ones for both biochemical bone markers $(p<0.000)$ Also, the percentage of osteocalcin change is $34.07 \%$, and the percentage of $\beta C T x$ change is $63,56 \%$ which exceeds values of least significant change (LSC), necessary to conclude that the difference is the effect of receiving bisphosphonate therapy

Table 4: Average values of tested laboratory parameters before the therapy and during it: osteocalcin (OC1 and OC2), $\beta C T x$ (CL1 and CL2) for 178 clinical participants receiving bisphosphonate therapy. 
Citation: Kovacev-Zavisic B, Icin T, Novakovic-Paro J, Medic-Stojanoska M, Mitrovic M, et al. (2013) Biochemical Bone Markers as Predictors of Excessive Bone Turnover Suppression during Osteoporosis Treatment with Bisphosphonates. J Mol Biomark Diagn S10: 001. doi:10.4172/2155-9929.S10-001

Page 4 of 7

\begin{tabular}{|l|l|l|l|l|l|l|}
\hline Group 1a (N=86) & $\bar{x}$ & Sd & Min & Max & Cv & Cl \\
\hline OC1 & 26.59 & 11.62 & 4.1 & 56.6 & 43.70 & $24.10-29.08$ \\
\hline CL1 & 428.59 & 232.63 & 18.8 & 1038.0 & 54.28 & $378.70-478.48$ \\
\hline Group 2a (N=92) & $\bar{x}$ & Sd & Min & Max & Cv & Cl \\
\hline OC1 & 36.64 & 11.89 & 13.0 & 78.9 & 32.44 & $34.18-39.10$ \\
\hline CL1 & 665.88 & 248.51 & 204.0 & 1290.0 & 37.32 & $614.41-717.36$ \\
\hline
\end{tabular}

Asymmetry and flattening values ranging from -.04 to .04 were not discussed Minimal (min) i maximal (max) biochemical markers of participants in group 1a indicate that values are within the expected range. Larger values of the coefficient of variation $(\mathrm{Cv})$ indicate that there is heterogeneity of Group 1a in terms of: OC (OC1) (43.70), CL1 (CL1) (54.28). Increased values of Skewness indicate that distribution is negatively asymmetric which means that distribution results curve tends toward larger values, i.e., there are larger values in comparison to normal distribution in: OC1 (OC1) (.28), CL1 (CL1) (.15). Negative values of Curtosis indicate that curve is flattened at: OC1 (OC1) (-.56), CL1 (CL1) (-.77). Distribution of values range mostly within normal distribution $(p)$ in: OC1 (OC1) (.22), CL1 (CL1) (.61)

Minimal (min) i maximal (max) biochemical markers of participants in Group 2a indicate that values are within the expected range. Larger values of the coefficien of variation $(\mathrm{Cv})$ indicate that there is heterogeneity of the Group 2a in terms of: OC1 (OC1) (32.44), CL1 (CL1) (37.32). Increased values of Skewness indicate that distribution is negatively asymmetric which means that distribution results curve tends toward larger values, i.e., there are larger values in comparison to norma distribution in: OC1 (OC1) (.43), CL1 (CL1) (.60). Larger values of Kurtosis indicate that curve has been elongated at: OC1 (OC1) (.44). Negative values of Kurtosis indicate that curve has been flattened at: CL1 (CL1) (-.05). Value distribution ranges mostly within normal distribution (p) at: OC1 (OC1) (.94), CL1 (CL1) (.57)

Table 5: Central and dispersive parameters and asymmetry and flattening measures of laboratory parameters during the therapy in groups $(\mathrm{N}=178)$.

\begin{tabular}{|c|c|c|c|}
\hline Analysis & $\mathbf{n}$ & $\mathbf{F}$ & $\mathbf{p}$ \\
\hline MANOVA & 2 & 23.666 & 0.000 \\
\hline discriminative & 2 & 25.122 & 0.000 \\
\hline
\end{tabular}

Table 6: The significance of differences between Group 1a and Group 2a with regard to biochemical bone markers before therapy (MANOVA).

\begin{tabular}{|c|c|c|c|}
\hline & $\mathbf{F}$ & $\mathbf{p}$ & c.dsc \\
\hline OC1 & 32.457 & .000 & .044 \\
\hline CL1 & 43.103 & .000 & .000 \\
\hline
\end{tabular}

c.dsc refers to coefficient of discrimination

Table 7: The significance of difference between Group 1a and Group 2a with regard to biochemical bone markers before therapy (discriminative analysis)

that pre-therapeutic values of both biochemical bone markers are lower in Group 1a in comparison to the Group 2a. That is represented by ellipses of confidence intervals shown on the graph 1 .

The line between groups of examinees with regard to biochemical bone markers is mathematically defined. If we add osteocalcin and $\beta C T x$ values before the therapy to the equation presented in the table 8 , we can conclude that patients having result $>0$, will have excessive suppression of bone resorption during the therapy, while patients having $<0$ will have desirable suppression of bone metabolism during the therapy. Homogeneity of groups is shown in table 9 .

\section{Excessive suppression of entire bone remodeling}

After these examinations, we have observed the patients (clinical participants) whose values of osteocalcin and $\beta \mathrm{CTx}$ during the bisphosphonate therapy have been below the bottom line of reference values (Group 1b) after three months. They could be said to have excessively suppressed total bone remodeling in comparison to other participants (Group 2b). Central and dispersive parameters and asymmetry and flattening measures of laboratory parameters during the therapy in groups are shown in table 10.

Based on values $\mathrm{p}=0.003$ (analysis MANOVA) and $\mathrm{p}=0.001$ for osteocalcin and $\mathrm{p}=0.006$ for $\beta \mathrm{CTx}$ (discriminative analysis) from tables 11 and 12 , we could conclude that significant statistical difference between groups of participants is such that pre-therapeutic values of both biochemical bone markers are lower in Group 1a in comparison to the Group 2a. That is represented by ellipses of confidence intervals shown on the graph 2.

The line between groups of participants with regard to biochemical bone markers is mathematically defined. If we add osteocalcin and $\beta C T x$ values before the therapy to the equation presented in the table 13 , we could conclude that patients having result $>0$, will have excessive suppression of entire bone remodeling during the therapy, while patients having $<0$ will have desirable suppression of bone metabolism, during the antiresorptive therapy. Homogeneity of groups is shown in table 14 .

\section{Discussion}

This study mainly indicates the fact already known that therapy of osteoporosis with bisphosphonates leads to suppression of bone metabolisms which is considered to be expected effect of this therapy. According to our results, the effect of therapy on the reduction of bone markers did not depend on the type (alendronate and ibandronate) or on doses of bisphosphonates ( $10 \mathrm{mg}$ on a daily basis, $70 \mathrm{mg}$ per week or $150 \mathrm{mg}$ on a monthly basis) which were used.

Bisphosphonate therapy caused expected and desirable suppression of bone metabolism and brought back the process of bone turnover onto the level of physiology in patients with initially increased bone metabolism. It has been shown many times that this suppression leads to significant increase of Bone Mineral Density (BMD) and reduces the risk of fracture in patients with pre-therapeutic increased bone

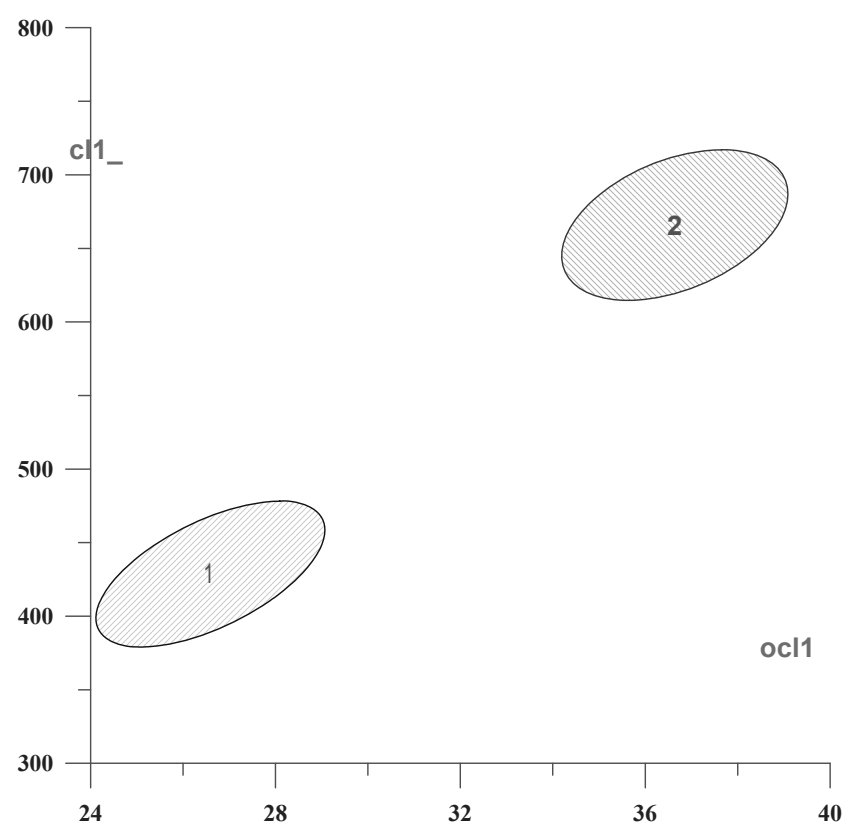

Group 1a (1); Group 2a (2);; OC1 (ocl1); CL1 (cl1)

$\mathrm{X}$-axis (horizontal axis) referring to OC1 (ocl1), and ordinate (vertical axis) referring to $\mathrm{CL} 1(\mathrm{cl} 1)$ are presented on the Graph 1

Graph 1: Ellipses (confidence intervals) referring to the groups of participants in OC1 i CL1. 
Citation: Kovacev-Zavisic B, Icin T, Novakovic-Paro J, Medic-Stojanoska M, Mitrovic M, et al. (2013) Biochemical Bone Markers as Predictors of Excessive Bone Turnover Suppression during Osteoporosis Treatment with Bisphosphonates. J Mol Biomark Diagn S10: 001. doi:10.4172/2155-9929.S10-001

Page 5 of 7

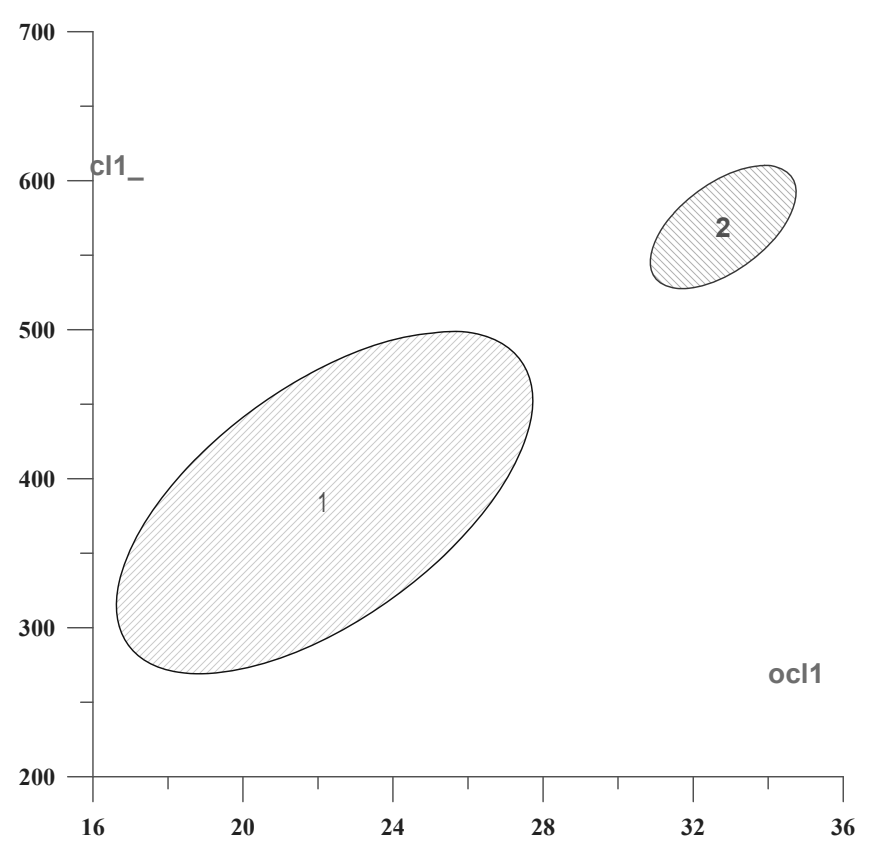

Group1b (1); Group 2b (2);: OC1 (oc/1); CL1 (cl1)

$\mathrm{X}$-axis (horizontal axis) referring to OC1 (ocl1), and ordinate (vertical axis) referring to $\mathrm{CL} 1$ ( $\mathrm{cl} 1)$ were presented on the Graph 2

Graph 2: Ellipses (confidence intervals) referring to groups of participants in OC1 i CL1.

metabolism [24,25]. However, previous studies of Andrea Giusti et al. [21] in 2010 along with studies of Tero Yli Kyyny [22] conducted in 2011, show us that excessive suppression of bone turnover can bring about undesirable effects of this therapy such as atypical fractures of femur. Excessive suppression of bone turnover leading to complete absence of active bone remodeling was immunohistochemically demonstrated by these studies in most patients with atypical femur fractures $[21,22]$.

In animal studies with alendronate, risedronate and incardronate during 1-3 years, higher levels of microdamage of trabecular bone of lumbar spine and cortical bone of ribs has been shown. These accumulations of microdamages were time and dose dependent [2628].

Having in mind that this microenvironment is expected to have reduced reparation ability of already existing micro damages as well as the accumulation of micro damages due to the time, undesirable effects of bisphosphonate therapy occur in cases of excessive suppressed bone metabolism $[29,30]$.

With treatment duration now extending into its second decade in some patients, it remains possible that over time the changes in material-level properties could override the structural-level benefits [31].

Based on the findings of our research, we deem that it is possible to predict which patients will have excessive suppression of bone metabolisms during the therapy based on the values of osteocalcin and $\beta C T x$ - before introducing bisphosphonate therapy. We also think that it is vital to predict whether there will be the occurrence of excessive suppression of bone resorption or of the whole process of bone remodeling.
We consider that patients, predicted to have desirable bone remodeling suppression also have to receive antiresorptive therapy of osteoporosis as the first line therapy (patients scored $<0$ in equation from tables 8 and 13).

It is possible that the patients with excessive suppression of

\begin{tabular}{|c|c|c|}
\hline$>0$ & $<0$ & Line equation \\
\hline Group1a & Group 2a & -.041 ocl1 $-.003 c / 1+2.983$ \\
\hline
\end{tabular}

Table 8: The line between groups of participants with regard to biochemical bone markers.

\begin{tabular}{|c|c|c|}
\hline & $\mathbf{n} / \mathbf{m}$ & homogeneity $\%$ \\
\hline Group 1a & $56 / 86$ & 65.12 \\
\hline Group 2a & $64 / 92$ & 69.57 \\
\hline
\end{tabular}

Table 9: Homogeneity of groups of participants with regard to biochemical bone markers.

\begin{tabular}{|c|c|c|c|c|c|c|}
\hline Group 1b (N=17) & $\bar{x}$ & Sd & Min & Max & Cv & Cl \\
\hline OC1 & 22.17 & 11.12 & 4.1 & 42.9 & 50.16 & $16.45-27.90$ \\
\hline CL1 & 383.92 & 230.20 & 18.8 & 852.3 & 59.96 & $265.53-502.31$ \\
\hline Group 2b (N=161) & $\bar{x}$ & Sd & Min & Max & Cv & $\mathrm{Cl}$ \\
\hline OC1 & 32.80 & 12.53 & 5.2 & 78.9 & 38.19 & $30.85-34.75$ \\
\hline CL1 & 568.90 & 266.33 & 63.6 & 1290.0 & 46.81 & $527.44-610.36$ \\
\hline
\end{tabular}

Asymmetry and flattening values ranging from -.04 to .04 were not discussed Minimal (min) i maximal (max) biochemical markers of participants in Group 1b indicate that values are within the expected range. Larger values of the coefficient of variation $(\mathrm{CV})$ indicate that there is heterogeneity of Group $1 \mathrm{~b}$ in terms of: OC1 (OC1) (50.16), CL1 (CL1) (59.96). Increased values of Skewness indicate that distribution is negatively asymmetric which means that distribution results curve tends toward larger values, i.e., there are larger values in comparison to normal distribution in: OC1 (OC1) (.11), CL1 (CL1) (.22). Negative values of Curtosis indicate that curve is flattened at: OC1 (OC1) $(-1.12), \mathrm{CL} 1$ (CL1) $(-.35)$.Value distribution range mostly within normal distribution (p) at: OC1 (OC1) (.80), CL1 (CL1) (.73)

Minimal (min) i maximal ( $\max$ ) biochemical markers of participants in Group $2 b$ indicate that values are within the expected range. Larger values of the coefficient of variation $(\mathrm{Cv})$ indicate that there is heterogeneity of Group $2 \mathrm{~b}$ in terms of: OC1 (OC1) (38.19), CL1 (CL1) (46.81). Increased values of Skewness indicate that distribution is negatively asymmetric which means that distribution results curve tends toward larger values, i.e., there are larger values in comparison to normal distribution in: OC1 (OC1) (.33), CL1 (CL1) (.35). Negative values of Curtosis indicate that the curve is flattened at: OC1 (OC1) (.08). Value distribution range mostly within normal distribution (p) at: OC1 (OC1) (.66), CL1 (CL1) (.88)

Table 10: Central and dispersed parameters and asymmetry and flattening measures of laboratory parameters during the therapy, done in groups. $(\mathrm{N}=178)$.

\begin{tabular}{|c|c|c|c|}
\hline Analysis & $\mathbf{n}$ & $\mathbf{F}$ & $\mathbf{p}$ \\
\hline MANOVA & 2 & 6.034 & 0.003 \\
\hline discriminative & 2 & 6.103 & 0.003 \\
\hline
\end{tabular}

Table 11: The significance between Group $1 \mathrm{~b}$ and Group 2b with regard to biochemical bone markers before therapy (MANOVA).

\begin{tabular}{|c|c|c|c|}
\hline & $\mathbf{F}$ & $\mathbf{p}$ & $\mathbf{c . d s c}$ \\
\hline OC1 & 11.280 & .001 & 0.027 \\
\hline CL1 & 7.593 & .006 & 0.000 \\
\hline
\end{tabular}

c.dsc refers to coefficient of discrimination

Table 12: The significance between Group $1 \mathrm{~b}$ and Group 2b with regard to biochemical bone markers before therapy (discriminative analysis).

\begin{tabular}{|c|c|c|}
\hline$>0$ & $<0$ & Line equation \\
\hline Group 1b & Group 2b & $\begin{array}{c}-.054 \text { ocl1 }-.001 \mathrm{cl1} \\
\mathbf{+ 2 . 0 7 5}\end{array}$ \\
\hline
\end{tabular}

Table 13: The line between groups with regard to biochemical bone markers. 
Citation: Kovacev-Zavisic B, Icin T, Novakovic-Paro J, Medic-Stojanoska M, Mitrovic M, et al. (2013) Biochemical Bone Markers as Predictors of Excessive Bone Turnover Suppression during Osteoporosis Treatment with Bisphosphonates. J Mol Biomark Diagn S10: 001. doi:10.4172/2155-9929.S10-001

Page 6 of 7

\begin{tabular}{|c|c|c|}
\hline & $\mathbf{n} / \mathbf{m}$ & homogeneity $\%$ \\
\hline Group 1b & $11 / 17$ & 64.71 \\
\hline Group 2b & $105 / 161$ & 65.22 \\
\hline
\end{tabular}

Table 14: Homogeneity of groups with regard to biochemical bone markers.

total bone remodeling will have accumulation of micro damages and eventually the occurrence of atypical fractures after receiving bisphosphonate therapy (Group 1b). At the same time, patients having normal or low values of biochemical bone markers before the therapy do not have enough active bone remodeling sites where bisphosphonates may bind and achieve its effect on increasing bone mineralization after introducing bisphosphonate therapy. We cannot expect significant increase of BMD and thereby the reduction of the fracture risk in these cases. These patients cannot have noteworthy benefits from bisphosphonate therapy. On the other hand, there is a potential risk that these patients will have the accumulation of undesirable effects.

Based on the results of this research, we can predict before introducing the therapy which patients will have excessive suppression of entire bone remodeling during bisphosphonate therapy (equation in table 13). These are mainly the patients whose initial values of biochemical bone markers are below the reference values or within its range before the therapy. We suggest these patients should be treated mainly with the stimulator of bone formation or with medications with dual mode of action (stimulating bone formation and inhibiting bone resorption).

On the grounds of this research results, predictions can be made about the patients who will have excessive suppression of bone resorption (Group 1a, equation in table 8) during the bisphosphonate therapy. We advise that apart from medications that stimulate bone formation or possibly medications with dual mode of action, antiresorptive therapy could also be the option for these patients.

There is a lack of data in the literature obtained from human studies. Also, there is little data on the effect of antiresorptive therapy in patients with low bone turnover.

The group of patients with low biochemical bone markers during antiresorptive therapy of osteoporosis should be additionally researched for the purpose of assessing the risk of fracture, the benefits of antiresorptive therapy for these patients and the evaluation of possible increase of harmful effects due to antiresorptive therapy.

\section{Conclusions}

Antiresorptive therapy with bisphosphonates leads to the reduction of bone resorption three months after it is being introduced. Values of biochemical bone markers during the therapy can be predicted depending on the initial values. We suggest that osteoporosis patients who are predicted to have excessive suppression of entire bone remodeling during bisphosphonate therapy, should be mainly treated with stimulators for bone formation or possibly with medications with dual mode of action (stimulation of bone formation and inhibition of bone resorption). Further research of low values of biochemical bone markers during the antiresorptive therapy is necessary for the purpose of assessing reductions of the fracture risk, benefits from antiresorptive therapy and evaluation of possible harmful effects of antiresorptive therapy.

\section{References}

1. (1993) Consensus Development Conference: Diagnosis, prophylaxis and treatment of osteoporosis. Am J Med 94: 646-650.

2. Kovacev Branka (1999) Patogenesis and diagnosing osteoporosis. 2nd
Yugoslav Rheumatology days 1999. Abstract book and original paperwork 23 30. (In Serbian)

3. NOF's Clinician's Guide for Prevention and Treatment of Osteoporosis. (2008) Bone Source.

4. (2000) Osteoporosis Prevention, Diagnosis, and Therapy. NIH Consens Statement 17: 1-36.

5. Hough S (2007) A rational approach to the treatment of osteoporosis. SA Fam Pract 49: 26-34.

6. Raisz LG (2005) Pathogenesis of osteoporosis: concepts, conflicts, and prospects. J Clin Invest 115: 3318-3325.

7. Saag KG, Shane E, Boonen S, Marin F, Donley DW, et al. (2007) Teriparatide or Alendronate in Glucocorticoid-Induced Osteoporosis. NEJM 357: 20282039

8. Miller PD, Delmas PD, Lindsay R, Watts NB, Luckey M, et al. (2008) Early Responsiveness of Women with Osteoporosis to Teriparatide After Therapy with Alendronate or Risedronate. J Clin Endocrinol Metab 93: 3785-3793.

9. Zhang S, Wright JE, Bansal G, Cho P, Uludag H (2005) Cleavage of Disulfide Linked Fetuin-Bisphosphonate Conjugates with Three Physiological Thiols. Biomacromolecules 6: 2800-2808.

10. Dimic A (2001) Bisphosphonates in osteoporosis treatment. Balneoclimatology 25: 21-25. (In Serbian)

11. Rosen CJ (2005) Clinical practice. Postmenopausal Osteoporosis, N Engl J Med 353: 595-603.

12. van Meurs JB, Dhonukshe-Rutten RA, Pluijm SM, van der Klift M, de Jonge $R$ et al. (2004) Homocysteine levels and the risk of osteoporotic fracture. N Eng J Med 350: 2033-2041.

13. Prodanovic N, Bozic B (2004) Clinical use of biochemical bone markers in osteoporosis. Balneoclimatology 28: 99-111. (In Serbian)

14. Srivastava AK, Vliet EL, Lewiecki EM, Maricic M, Abdelmalek A, et al (2005) Clinical Use of Serum and Urine Bone Markers in the Management of Osteoporosis. Curr Med Res Opin 21: 1015-1026.

15. Seibel MJ (2005) Biochemical Markers of Bone Turnover: Part I: Biochemistry and variability. Clin Biochem Rev 26: 97-122.

16. Seibel MJ (2006) Biochemical Markers of Bone Turnover Part II: Clinica Applications in the Management of Osteoporosis. Clin Biochem Rev 27: 123

17. Rosen HN, Moses AC, Garber J, lloputaife ID, Ross DS, et al. (2000) Serum CTX: A New Marker of Bone Resorption That Shows Treatment Effect More Often Than Other Markers Because of Low Coefficient of Variability and Large Changes with Bisphosphonate Therapy. Calcif Tissue Int 66: 100-103.

18. Mashiba T, Hirano T, Turner CH, Forwood MR, Johnston CC, et al. (2000) Suppressed Bone Turnover by Bisphosphonates Increases Microdamage Accumulation and Reduces Some Biomechanical Properties in Dog Rib. Bone Miner Res 15: 613-620.

19. Odvina CV, Zerwekh JE, Rao DS, Maalouf N, Gottschalk FA, et al. (2005) Severely Suppressed Bone Turnover: A Potential Complication of Alendronate Therapy. J Clin Endocrinol Metab 90: 1294-1301.

20. Visekruna M, Wilson D, McKiernan FE (2008) Severely Suppressed Bone Turnover and Atypical Skeletal Fragility. J Clin Endocrinol Metab 93: 29482952.

21. Giusti A, Hamdy NA, Papapoulos SE (2010) Atypical fractures of the femu and bisphosphonate therapy: A systematic review of case/case series studies. Bone 47: 169-180.

22. Yli-Kyyny T (2011) Bisphosphonates and Atypical Fractures of Femur. J Osteoporos 2011: 754972.

23. State expert committee to guide the development and implementation of clinica practice guidelines. Osteoporosis: a national guide for doctors in primary care. Belgrade, Faculty of Medicine, University of Belgrade, Serbia, November 2004 (In Serbian)

24. Allen MR, Burr DB (2011) Bisphosphonate effects on bone turnover microdamage, and mechanical properties: What we think we know and what we know that we don't know. Bone 49: 56-65.

25. Bauer DC, Garnero P, Hochberg MC, Santora A, Delmas P, et al. (2006) 
Citation: Kovacev-Zavisic B, Icin T, Novakovic-Paro J, Medic-Stojanoska M, Mitrovic M, et al. (2013) Biochemical Bone Markers as Predictors of Excessive Bone Turnover Suppression during Osteoporosis Treatment with Bisphosphonates. J Mol Biomark Diagn S10: 001. doi:10.4172/2155-9929.S10-001

Pretreatment levels of bone turnover and the antifracture efficacy of alendronate: the fracture intervention trial. J Bone Miner Res 21: 292-299.

26. Mashiba T, Turner CH, Hirano T, Forwood MR, Johnston CC, et al. (2001) Effects of suppressed bone turnover by bisphosphonates on microdamage accumulation and biomechanical properties in clinically relevant skeletal sites in beagles. Bone 28: 524-531

27. Allen MR, Iwata K, Phipps R, Burr DB (2006) Alterations in canine vertebra bone turnover, microdamage accumulation, and biomechanical properties following 1-year treatment with clinical treatment doses of risedronate or alendronate. Bone 39: 872-879.

28. Komatsubara S, Mori S, Mashiba T, Li J, Nonaka K, et al. (2004) Suppressed bone turnover by long-term bisphosphonate treatment accumulates microdamage but maintains intrinsic material properties in cortical bone of dog rib. J Bone Miner Res 19: 999-1005.

29. Li J, Mashiba T, Burr DB (2001) Bisphosphonate treatment suppresses not only stochastic remodeling but also the targeted repair of microdamage. Calcif Tissue Int 69: 281-286

30. Stepan JJ, Burr DB, Pavo I, Sipos A, Michalska D, et al. (2007) Low bone mineral density is associated with bone microdamage accumulation in postmenopausal women with osteoporosis. Bone 41: 378-385.

31. Allen MR, Burr DB (2007) Mineralization, microdamage, and matrix: How bisphosphonates influence material properties of bone. BoneKEy-Osteovision 4. $49-60$

This article was originally published in a special issue, Bone Biomarkers handled by Editor. Dr. Yi Wang, Cornell University, USA 